\title{
Study on Cultivation of Core Literacy of Nursing Students in Traditional Chinese Medicine Colleges Under the Background of Healthy China
}

\author{
Jun $\mathrm{Li}^{1}$, Zhenyu $\mathrm{Hu}^{2 *}$, Sijia Luo ${ }^{3}$, Quan $\mathrm{Xia}^{4}$ \\ ${ }^{1}$ Jiangxi University of Traditional Chinese Medicine, Nanchang, Jiangxi, China \\ ${ }^{2}$ Jiangxi University of Traditional Chinese Medicine, Nanchang, Jiangxi, China \\ ${ }^{3}$ Jiangxi University of Traditional Chinese Medicine, Nanchang, Jiangxi, China \\ ${ }^{4}$ Jiangxi University of Traditional Chinese Medicine, Nanchang, Jiangxi, China \\ *Corresponding author. Email:491673015@qq.com.
}

\begin{abstract}
With the rapid development of social economy, people's pursuit of the standard of their own health has changed from simply "eating well and wearing warm clothing" to having a high -quality life and a healthy and strong body. Since the 19th National Congress of the Communist Party of China, China has raised the concept of "healthy China" to the national strategic level for the first time, giving priority to people's health. As one of the cultures with a long history in China, Chinese medicine culture is also an important part of China's medical and health system up to now. As a key link in Chinese medicine technology, Chinese medicine nursing plays an important role in national health, especially the health management of the elderly.
\end{abstract}

Keywords: Healthy Chinese, Traditional Chinese Medicine Nursing, Core Literacy

\section{INTRODUCTION}

Since the 21 st century, with the continuous development of economy and society, possessing hightech talents has become an important factor to improve international competitiveness. Therefore, education increasingly determines the future development direction and achievements of the country. For our country, education should not only pursue "quantity" but also "quality". The concept of core literacy was first put forward by the Council of the European Union in 2002. For our country, the concept of core literacy was put forward late, and it was not until 2016 that Beijing Normal University released the specific contents and requirements for Chinese students to develop core literacy. In the future, China is required to educate a new generation of young people in three aspects: cultural foundation, independent development and social participation, so as to cultivate an all-round development person for the country. On the basis of these three aspects, the core literacy is further subdivided into six specific literacy, namely, humanistic heritage and scientific spirit, learning to learn and live healthily, responsibility and practical innovation, hoping to cultivate people with the necessary character and ability for lifelong development and social development in the new era ${ }^{[1]}$.

\section{DEVELOPMENT STATUS OF CORE LITERACY OF TRADITIONAL CHINESE MEDICINE NURSING}

In March 2011, according to the new edition of professional classification catalogue issued by the Ministry of Education, the separation of nursing discipline from clinical medicine into an independent first-class discipline has further promoted the development of nursing discipline, and at the same time expressed the public's increasing demand for health, and the need for high-quality and high-level nursing talents and services. Traditional Chinese medicine is a traditional medicine with a long history in our country. Its main idea of "preventing diseases" is compatible with nursing. The combination of the two has produced the major of traditional Chinese medicine nursing. This major is a discipline under the guidance of the basic theory of traditional Chinese medicine. It teaches students nursing operation techniques and various nursing methods in traditional Chinese medicine ${ }^{[2]}$.

For students of this major, the cultivation of core literacy not only requires students to master professional 
skills and professional knowledge, but also requires them to improve the work skills needed in future work, such as the interpersonal communication ability to communicate with patients at work, and the ability to independently improve professional skills in spare time ${ }^{[3]}$. Through an investigation on the current situation of nursing core literacy of 370 nursing undergraduates in a medical college, it can be seen that, at present, there is a phenomenon in colleges and universities that the propaganda of core literacy is not high and the awareness of students is low. Through the questionnaire research, it shows that the propaganda of developing core literacy in colleges and universities is insufficient, and the awareness among students is not high. College education is a key step before nursing students set foot on work, it includes not only book education in learning but also clinical practice in actual combat. The two are closely linked. Only when education is done well can we lay a solid foundation for the cultivation of students' core literacy and provide help for the society to cultivate allround development of nursing talents.

\section{PROBLEMS ENCOUNTERED IN THE DEVELOPMENT OF TCM NURSING}

\subsection{The problem of population aging is serious}

According to international standards, a country's population over 65 years old accounts for more than $7 \%$, which means entering an aging society. For China, China entered an aging society as early as 2000 , when the elderly population over 65 years old exceeded 88 million, accounting for almost $7 \%$ of the total population. By the end of 2019, this data continued to increase, and the number of elderly people aged 65 and above reached 176 million, accounting for about $12.6 \%$ of the total population ${ }^{[4]}$. According to speculation, China's aging population will reach at least 300 million by 2025 , which means that China will officially enter an aging society from an aging society. It can be seen that compared with the situation that it takes at least hundreds of years for western countries to enter an aging society, China's aging population is characterized by large scale and fast growth.

\subsection{Shortage of elderly care population}

The increase of the elderly population requires adequate supply of corresponding old-age services, oldage facilities and nursing staff. Further requirements are put forward in the Outline of Nursing Development Plan released in 2016, It is required that by 2020, the number of registered nurses in China should reach 4.45 million, the average number of nurses per 1,000 people in China should reach 3.14 , the number of practicing doctors should be 1.25 times that of registered nurses, and at least 6 nurses should be equipped for every 10 beds in tertiary general hospitals. According to this outline, it is predicted that China needs to train about 200,000 nurses at all levels every year. In addition, there is a shortage of talents specializing in geriatric care in China, according to statistics, there are only about 300,000 professional nursing staff in the existing old-age care institutions, of which only about 40,000 hold professional qualification certificates. Among them, there are fewer professionals specialized in traditional Chinese medicine nursing. Compared with China's huge old-age care market, the number of these professionals is far from enough.

\subsection{There are few colleges and universities offering nursing majors}

The Ministry of Education and the National Health and Health Commission have included nursing majors in the national shortage of talents. The number of nursing professionals trained by Chinese colleges and universities every year cannot meet the market demand, and the current nursing education is biased towards Western medicine, and the nursing education of Chinese medicine is relatively lacking ${ }^{[5]}$. There are 23 colleges and universities of traditional Chinese medicine in China, but these colleges and universities mainly train talents of traditional Chinese medicine, and the enrollment of nursing major is far lower than that of traditional Chinese medicine major. On the contrary, most colleges and universities offering TCM nursing majors think that higher vocational and technical secondary schools are the main ones, which leads to the low level and weak foundation of TCM nursing talents. Secondly, there is less nursing education at the master's or even doctoral level. According to the statistics of China Postgraduate Enrollment Information Network, among the enrollment information of various universities in China, there are less than 50 nursing colleges and universities at the master's level, and even fewer at the doctoral level, less than 20 .

\section{FUTURE DEVELOPMENT PATH OF TRADITIONAL CHINESE MEDICINE NURSING}

\subsection{Actively construct the training system of "university-hospital" cooperation with Chinese medicine characteristics}

For students majoring in traditional Chinese medicine nursing, classroom education and clinical education are essential parts of cultivating core literacy ability in the learning process. For classroom theoretical study, it is very necessary to build an excellent team of teachers of traditional Chinese medicine nursing specialty for colleges and universities offering traditional Chinese medicine nursing specialty. Teachers with strong professional knowledge and high theoretical level should be screened out, and relevant courses should be set up according to the specific requirements of cultivating core 
literacy to improve students' professional literacy. With the goal of cultivating all-round people, Add professional courses such as geriatric nursing and community nursing to the curriculum, and increase the curriculum of humanities and social sciences for nursing majors, To improve the interpersonal communication ability of nursing students at work, teachers can use heuristic, PBL teaching, flip class, discussion, research learning and other diversified teaching methods in the classroom to cultivate students' team cooperation ability, critical thinking ability and innovative ability. For clinical teaching in the practice process, this is an opportunity for nursing students to exercise themselves and test their learning ability. Here, teachers need to train students' core literacy ability in the training process, mobilize students' enthusiasm for autonomous learning, listen more, think more and ask more, and change from passive learning to active learning.

In addition, under the background of aging, we should pay attention to strengthening nursing education for middle-aged and elderly people in the cultivation system Increase students' sense of identity with elderly nursing work, guide nursing students to broaden their employment ideas and change their traditional employment directions and values, encourage them to engage in the elderly care industry. For example, special courses such as Chinese medicine geriatric health care and geriatric psychology are set up in school theoretical education to increase students' understanding and learning of nursing knowledge for the elderly. The school encourages nursing students to go to the community to participate in volunteer service on rest days, give full play to their professional expertise, and recommend students to special elderly departments during hospital practice, so that they can rationally understand elderly nursing work in the specific practice process.

\subsection{Strive to establish a professional evaluation method for core literacy}

The concept of core literacy was first put forward by the European Union. They not only defined the current situation and specific concepts of core literacy, but also proposed to establish an evaluation system of core literacy. The Outline of Health Development promulgated by our country also puts forward that it is necessary to constantly improve the setting of nursing posts in hospitals and establish a perfect professional nursing post training system. For professional nurses, not only pre-job training is necessary, but also, they need to constantly improve themselves in the process of work. Training and self-improvement [6]. The specific connotation of core literacy covers all kinds of qualities and abilities that nurses should possess. A set of perfect core literacy evaluation system can be established to formulate training standards for clinical specialist nurses, improve their abilities in their work, and enable patients to enjoy better nursing services. At the same time, the specific core literacy evaluation system can set selfdevelopment goals for nursing students at all levels, and determine the level they need to reach according to their own abilities. The construction of such index system can promote the development of nursing specialty in China and point out the direction for future nursing specialty.

\section{CONCLUSION}

At present, the development of the concept of core literacy in our country is still in its infancy, but as a theme involved in the field of education all over the world, improving the core literacy system is an important direction for the development of education in our country in the future. The combination of the content of core literacy and the training objectives of TCM nursing specialty indicates what kind of talents should be trained and how to train talents in TCM nursing in the future. With the implementation of the Healthy China Strategy and the deepening of China's aging level, it requires Chinese universities and social organizations to make continuous efforts to cultivate talents and meet the rising demand for nursing talents.

\section{REFERENCES}

[1] Core Literacy Research Group. Chinese students develop core literacy [J]. China Journal of Education, 2016 (10): 1-3.

[2] ZHANG Bo-ya. Development and prospect of TCM nursing $[\mathrm{J}]$. China Health Care Nutrition, 2017, 27 (20): 214. DOI: 10.3969/j.issn.1004-7484. 2017.20.307.

[3] Ma Junqing. Investigation on core literacy of nursing undergraduates and countermeasures $[\mathrm{J}]$. Medical Education Research and Practice, 2018, 26 (2): 309313. DOI: 10.13555/j. cnki. c.m.e.2018.02.035.

[4] Ni Enjie, Lu Jin.A new model to solve the problem of providing for the aged-building a smart home care platform [J]. Operation and Management, 2021, (05): $115-118$

[5] Dong Xue, Bu Xuejiao, Li Songze, Yan Li. Research progress on training and education of TCM nursing professionals [J]. Abstracts of World Latest Medical Information, 2017, 17 (23): 256.

[6]. Outline of China's Nursing Development Plan (20112015) [J]. Contemporary Nurses (Shanghai Xunkan), 2012, (06): 9-12. 